\title{
Cultivo de Ceratocystis (Ophiostoma) Ulmi (Buism.) Moreau en MEDIO LÍQUido, E INOCULACIÓN
}

\author{
María Laura SALVADOR NEMOZ, Andrés MARTINEZ DE AZAGRA, Juan \\ Andrés ORIA DE RUEDA, \\ María Eugenia GARCÍA NIETO, \& Luis GIL SÁNCHEZ
}

RESUMEN: En el presente trabajo se ensaya la técnica de inoculación de Ceratocystis (Ophiostoma) ulmi (Buism.) Moreau, en olmos procedentes de semilla y se observa la respuesta de las plantas a la enfermedad.

Palabras clave: Inoculación, Ceratocystis (Ophiostoma) ulmi, Grafiosis.

SUMMARY: The methodology to obtain yeastlike phase and to evaluate resistance to Ceratocystis (Ophiostoma) ulmi (Buism.) Moreau, was tested using aggressive and nonaggressive strains.

Key words: Inoculation, Ceratocystis (Ophiostoma) ulmi, Dutch Elm Disease.

\section{INTRODUCCIÓN}

La grafiosis es una enfermedad mortal de los olmos que debe ser atacada por todos los caminos posibles; uno de ellos es la búsqueda de ejemplares resistentes para su posterior difusión.

Esta vía consiste en desarrollar, en base a un programa de mejora, individuos con dicha característica como resultado de su existencia en las poblaciones naturales, o a través de cruzamientos controlados.

En España se ha iniciado un programa de mejora que se apoya en las experiencias obtenidas en otros centros de investigación (Ipinza \& Gil, 1988).

Evaluar la resistencia a la enfermedad de los individuos seleccionados es un aspecto básico, para ello se debe introducir el patógeno en el hospedante y observar su respuesta. 


\section{OBJETIVOS}

Se plantea ensayar la técnica de inoculación como tal, en olmos procedentes de semilla. En base a esto se fijan los siguientes objetivos.

1 - Desarrollo de la metodología necesaria para obtener Ceratocystis (Ophiostoma) ulmi en su fase de levadura, utilizando el medio nutritivo de Zentmyer modificado por Tchernoff (Brasier 1981a).

2 - Realizar un conteo de levaduras para determinar la evolución del crecimiento del hongo en el tiempo y obtener la concentración del inóculo.

3 - Comprobar los postulados de Koch a partir de inoculaciones con una cepa agresiva.

4 - Observar la patogenicidad de las cepas utilizadas.

\section{MATERIAL Y MÉTODOS}

INOCULO. Para realizar esta experiencia se contó con dos aislamientos propios de $C$. (Ophiostoma) ulmi, uno ALM 18 «no agresivo» de Almazán, Soria; con fecha de muestreo del 18 de julio de 1986, tasa de crecimiento 2,7 mm/día, de aspecto céreo, agregado y concéntrico; con tipo de unión B. El otro, Pueblas «agresivo» de Las Pueblas, Madrid; con fecha de muestreo del 6 de agosto de 1987, tasa de crecimiento 4,6 mm/día, de morfología aérea y petaloide; su tipo de unión es A.

PLANTAS. Se utilizaron 8 plantas de híbridos de Ulmus glabra y U. minor (s.1.) de 4 años de edad, contenidos en maceta y cultivados en condiciones ambientales naturales.

METODOLOGÍA. Se cultiva la cepa no agresiva en medio líquido de Zentmyer con modificación de Tchernoff utilizando un agitador durante 72 horas a $30^{\circ} \mathrm{C}$. Para la cepa agresiva la metodología es idéntica a excepción de la temperatura de agitación que es de $22^{\circ} \mathrm{C}$.

A lo largo de este período se hace el recuento de levaduras a diferentes intervalos de tiempo: intervalos de 1 hora entre las 0 y $12 \mathrm{~h}$ de comenzado el ensayo, intervalos de 2 horas entre las 24 y 36 h, e intervalos de 12 horas a partir de las $48 \mathrm{~h}$. (lámina 1: A).

El procedimiento de inoculación se hace por inyección de una gota del cultivo líquido del patógeno en la horcadura de la rama donde previamente se ha realizado un corte con escalpelo teniendo la precaución de no llegar al cambium.

Al cabo de 22 días se toman dos muestras de los olmos inoculados con la cepa agresiva Pueblas, $3 \mathrm{~cm}$ por arriba y por debajo del punto de inoculación (lámina 1: B). Estas se colocan en agar para evidenciar la presencia del hongo y comprobar los postulados de Koch. 


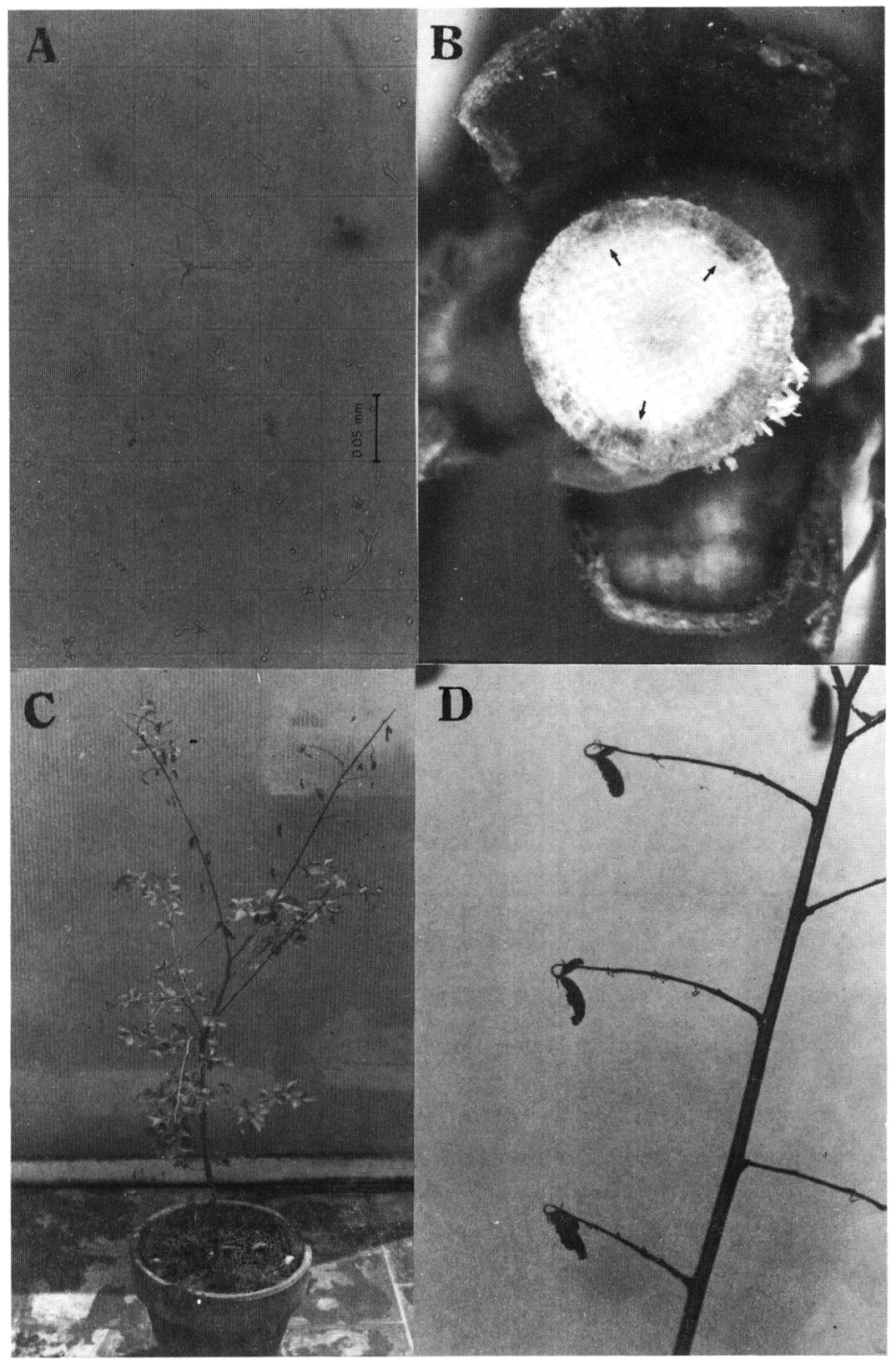

Lámina 1. A: Detalle de la cámara de recuento de levaduras. B: Síntomas en ramas de olmo, del que se reaisla C. (Ophiostoma) ulmi. C: Síntomas de grafiosis agresiva. D: Síntoma típico de grafiosis agresiva en forma de «J» invertida. 


\section{RESULTADOS Y CONCLUSIONES}

La concentración obtenida en medio líquido alcanza el valor de $10^{5}$ esporas $/ \mathrm{ml}$ en el tercer día de agitación y se mantiene en este valor. Se considera que se ha estabilizado el crecimiento en una concentración adecuada para la inoculación (Schreiber \& Stipes 1967; Green, Guries \& Smalley 1985) (Tabla I, Gráfico I).

\begin{tabular}{ccc}
\hline Hora & Concentración (esp./ml) & Log concentración \\
\hline $11 \mathrm{~h}$ & $8,5 \times 10^{3}$ & 3,93 \\
$12 \mathrm{~h}$ & $9,4 \times 10^{3}$ & 3,97 \\
$13 \mathrm{~h}$ & $9,7 \times 10^{3}$ & 3,99 \\
$14 \mathrm{~h}$ & $1,1 \times 10^{4}$ & 4,04 \\
$15 \mathrm{~h}$ & $1,4 \times 10^{4}$ & 4,15 \\
$16 \mathrm{~h}$ & $1,6 \times 10^{4}$ & 4,20 \\
$17 \mathrm{~h}$ & $1,8 \times 10^{4}$ & 4,25 \\
& & 4,38 \\
$11 \mathrm{~h}$ & $2,4 \times 10^{4}$ & 4,50 \\
$13 \mathrm{~h}$ & $3,2 \times 10^{4}$ & 4,58 \\
$15 \mathrm{~h}$ & $3,8 \times 10^{4}$ & 4,78 \\
$17 \mathrm{~h}$ & $6,1 \times 10^{4}$ & 5,20 \\
\hline $11 \mathrm{~h}$ & $1,6 \times 10^{5}$ & 5,20 \\
$18 \mathrm{~h}$ & $1,6 \times 10^{5}$ & 5,20 \\
\hline $12 \mathrm{~h}$ & $1,6 \times 10^{5}$ & \\
\hline
\end{tabular}

TABLA I. Crecimiento de C. (Ophiostoma) ulmi en medio líquido.

La técnica de inoculación que nos ha resultado más eficaz ha sido el goteo con jeringuilla sobre el corte con escalpelo.

Se verifica que el aislamiento Pueblas, y su reaislamiento obtenido tras la inoculación, presentan ambos las mismas características morfológicas y de crecimiento que el aislamiento primario.

La distinta patogenicidad de las dos cepas se observa en una aparición de los síntomas de la grafiosis a los 30 - 32 días en las plantas inoculadas con ALM 18, y a los 18 - 20 días en aquellas inoculadas con Pueblas.

Aunque ninguno de los olmos presenta resistencia a la enfermedad, sí se observan diferencias en su recuperación. Para el caso de las infecciones con ALM 18 se aprecia una recuperación de los olmos a partir de 40 - 45 días después de la aparición de los síntomas. Las infecciones con Pueblas no muestran signos de recuperación a los 60 días de aparición de los mismos. (lámina 1: D). 


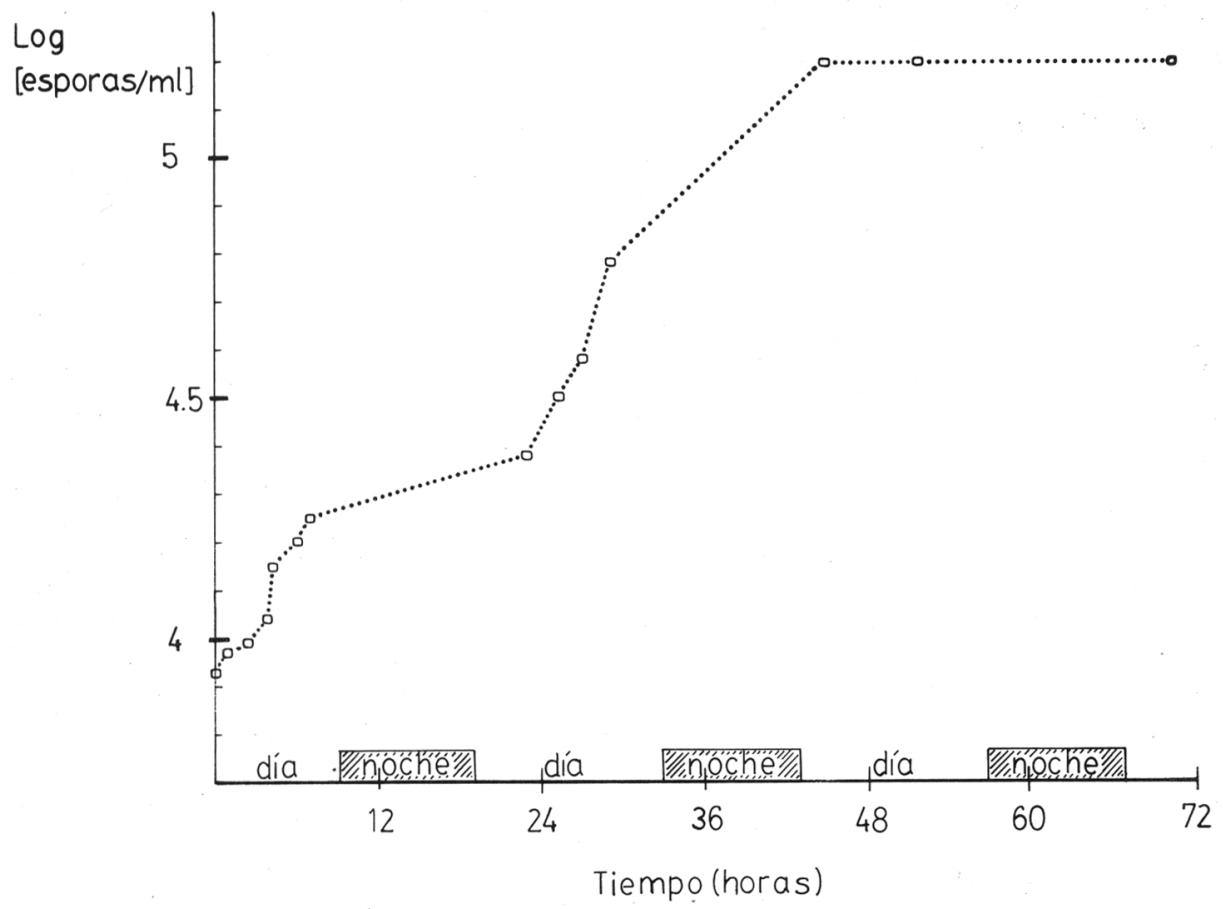

Gráfico I Crecimiento de C. (Ophiostoma) ulmi en medio líquido.

\section{BIBLIOGRAFÍA}

BRASIER, C. -1981a- Appendix II Laboratory Investigation of Ceratocystis ulmi. En «Compendium of Elm Disease» (R.J. Stipes \& R.J. Campana, Eds) pp 76-79. American Phytopathological Society, St. Paul, Minnesota.

GREEN, C., R. GURIES \& E. SMALLEY -1985- Early screening of elms for resistance to Ceratocystis ulmi. Plant Disease, 69:60-69.

IPINZA CARMONA, R. \& L. GIL SANCHEZ -1988- Mejora genética de los olmos frente a la Grafiosis. En: Mejora Genética de especies arbóreas forestales (Pardos J.A. Editor). Fundación Conde del Valle de Salazar, Madrid. pp: 394-410.

SCHREIBER, L. \& R. STIPES -1967- The effect of inoculum spore concentration on the development of foliar symptoms of Dutch Elm Disease. Phytopathological Notes pp: 1269.

(Aceptado para su publicación en Junio de 1.990)

Dirección de los autores: M.L. SALVADOR NEMOZ, J.A. ORIA DE RỤEDA, M.E. GARCÍA-NIETO \& L. GIL SÁNCHEZ: Departamento de Silvopascicultura. Escuela Técnica Superior Ingenieros de Montes. Ciudad Universitaria s/n 28040. Madrid. A. MARTINEZ DE AZAGRA: Departamento de Ciencias y Tecnología Agrarias. Escuela Universitaria Politécnica Agraria. Avda. de Madrid, 57: 34071. Palencia. 
